\title{
Interplay of QCD and EW corrections and precision physics at hadron colliders.
}

\author{
Alessandro Vicini* \\ Tif lab, Dipartimento di Fisica dell'Università di Milano and INFN Sezione di Milano, Via \\ Celoria 16, 20133 Milano (Italy) \\ E-mail: alessandro.viciniemi.infn.it
}

The interplay between QCD and EW corrections, relevant at a hadron collider for the highprecision measurement of EW parameters, is presented, with a brief overview of some currently open questions.

The European Physical Society Conference on High Energy Physics

5-12 July, 2017

Venice

${ }^{*}$ Speaker. 


\section{Introduction}

An important part of the LHC physics programs concerns the study of the mechanism of spontaneous breaking of the electroweak (EW) symmetry: the discovery of the Higgs boson and the study of its properties, the searches for anoumalous tri- and quadrilinear gauge boson couplings, the determination of the gauge boson properties and the precision tests of the Standard Model (SM) are important chapters of this physics program.

In the study of electroweak processes at a hadron collider the strong and eletroweak interactions are naturally entangled. The inclusive hadron-level cross section of scattering processes with large momentum transfer can be written, according to the factorization theorems, as the convolution of collinear proton parton density functions (PDFs) with the cross section of the scattering of partons, elementary constituents of the proton, in the desired final state $Y$ plus additional particles collectively represented by $X$.

$$
\sigma(p p \rightarrow Y+X)=\sum_{i, j} \int_{0}^{1} d x_{1} d x_{2} f_{i}^{I}\left(x_{1}\right) f_{j}^{I I}\left(x_{2}\right) \hat{\sigma}(i j \rightarrow Y+X)
$$

The partonic cross section $\hat{\sigma}(i j \rightarrow Y+X)$ can be expanded as a double series in the two couplings $\alpha$ and $\alpha_{s}$, respectively the fine structure and the strong coupling constants. The perturbative evolution of the PDFs, according to the DGLAP equations, is driven by a kernel which contains, beside the QCD ones, also the QED splitting functions. It is thus clear that mixed QCDxEW contributions affect all the elements contributing to any cross section to all perturbative orders and their consistent evaluation is needed to improve the precision of the theoretical predictions. In the following Sections we briefly review the open issues concerning the different parts of the theoretical predictions.

\section{Perturbative corrections at parton level}

The production of a pair of leptons, each with large transverse momentum, the so called DrellYan (DY) process, is one of the most relevant processes for EW precision measurements at the LHC, like the determination of the masses and decay widths of $W$ and $Z$ bosons and of the weak mixing angle. The precision goals of the measurements by the LHC experiments [1,2], with the error on some quantities like the $W$ boson mass at the $\mathscr{O}\left(10^{-4}\right)$ level, are very challenging for any theoretical prediction and many effects, considered so far as subleading, need to be carefully scrutinized to keep the theoretical uncertainties under control at the necessary level. This is the case for instance of the mixed QCD-EW corrections, starting from those of $\mathscr{O}\left(\alpha \alpha_{s}\right)$ with respect to the LO process.

The size of the first order EW corrections to the DY processes depends on the observable under study: while quantities whose definition is fully inclusive over the QED real radiation typically receive corrections of $\mathscr{O}(\alpha) \sim 1 / 137$, kinematical distributions of observables defined in terms of only the final state leptons momenta may receive a logarithmic enhancement due to soft/collinear final state QED emissions, with effects that can reach the $\mathscr{O}(10 \%)$ level in some specific phasespace corners. Analogous comments can be formulated for the QCD corrections, which receive, beside a large global K-factor, a logarithmic enhancement for all those observables sensitive to the 
details of initial state QCD radiation. The hierarchy between the purely QCD and EW corrections can be naively formulated by comparing the size of the coupling constants evaluated at the $Z$ boson mass scale, with $\alpha_{s}\left(m_{Z}\right) / \alpha\left(m_{Z}\right) \sim 15$ and $\alpha_{s}^{2}\left(m_{Z}\right) / \alpha\left(m_{Z}\right) \sim 1.8$. This hierarchy is roughly respected for all the observables whose definition is inclusive with respect to the emission of additional radiation, but it can be modified for exclusive quantities, when a logarithmic enhancement is present. A detailed classification of the size of radiative corrections to DY processes at NLO and beyond can be found in Ref. [3].

The absence of an exact calculation of the $\mathscr{O}\left(\alpha \alpha_{s}\right)$ corrections to the DY processes, valid through the whole DY phase space, leaves open a systematic discussion about the precise size of these effects. The exact evaluation, in pole approximation, of the $\mathscr{O}\left(\alpha \alpha_{s}\right)$ corrections to single gauge boson production $[4,5,6]$ allowed the explicit check, at the gauge boson resonance, of some approximations already available in the literature. An important caveat applies to the possible estimate of the size of $\mathscr{O}\left(\alpha \alpha_{s}\right)$ effects from the comparison of an additive and a factorized combinations of existing NLO-QCD and NLO-EW results: defining $\delta_{\alpha}$ and $\delta_{\alpha_{s}}$ the relative effect, with respect to the LO result $\sigma_{0}$, in one bin of one observable, of NLO-EW and NLO-QCD corrections, one can consider $\sigma_{a d d}=\sigma_{0}\left(1+\alpha \delta_{\alpha}+\alpha_{s} \delta_{\alpha_{s}}\right)$ and $\sigma_{f a c}=\sigma_{0}\left(1+\alpha \delta_{\alpha}\right)\left(1+\alpha_{s} \delta_{\alpha_{s}}\right)$ so that the difference $\sigma_{f a c}-\sigma_{a d d}=\alpha \alpha_{s} \delta_{\alpha} \delta_{\alpha_{s}}$, dubbed naive factorization, provides an estimate of the $\mathscr{O}\left(\alpha \alpha_{s}\right)$ corrections. In Refs. $[4,5,6]$ it is shown how this ansatz, compared to the exact calculation in pole approximation, fails in the description of the $\mathscr{O}\left(\alpha \alpha_{s}\right)$ corrections to the lepton transverse momentum distribution or to the lepton-pair invariant mass distribution in the neutral current DY process, while it provides an acceptable description of the effects on the lepton-pair transverse mass distribution in the charged current DY process. The failure of the naive factorization ansatz sounds contradictory with the fact that at the gauge boson resonance, the dominant contribution to mixed QCDxEW corrections is due to factorizable terms with a QCD corrections to the initial state and an EW correction dominated by QED final state radiation off the leptons. The non trivial point of these corrections is related to the kinematical interplay between QCD and EW effects, due to the structure of the cross section with the convolution of final state corrections over the kernel of the initial state ones. Once the full kinematics is properly taken into account, the correct size of the corrections, as given from the exact calculation in pole approximation, can be reproduced.

\section{Mixed QCDxEW corrections and the hadron-level cross section}

The relevance of a correct treatment of the radiation kinematics at exclusive level has been checked with the Monte Carlo event generator POWHEG [7, 8] with NLO-(QCD+EW) accuracy on the total cross section, matched with both a QCD and a QED Parton Shower (PS). The impact on the determination of the $W$ boson mass, extracted from the lepton-pair transverse momentum distribution and due to $\mathscr{O}\left(\alpha \alpha_{s}\right)$ has been estimated with POWHEG to be $\Delta m_{W}=-16 \pm 3 \mathrm{MeV}$, to be compared with the $\Delta m_{W}=-14 \mathrm{MeV}$ of the exact calculation in pole approximation.

Theoretical progress to achieve a complete prediction of the $\mathscr{O}\left(\alpha \alpha_{s}\right)$ corrections to the DY process requires the evaluation of the cross section at NNLO, with the new technical challenge due to the presence of new classes of two-loop integrals, like those described in Refs.[14, 15], where few internal lines are massive; the presence of several energy scales in the process requires the introduction of new classes of functions to provide an analytical representation of the result. 
In Ref.[9] a detailed analysis of several classes of purely EW and mixed QCDxEW corrections and of their corresponding impact on the determination of the $W$ boson mass. has been performed, relying on their implementation in the event generators HORACE and POWHEG. This study quantifies the large size of the $\mathscr{O}\left(\alpha \alpha_{s}\right)$ corrections for example in the case of the single charged lepton transverse momentum distribution. A strong dependence on the modelling of QED final state radiation (FSR) is observed when a leading logarithms (LL) accurate tool is applied on top of a given QCD prediction, as it is the case for instance of the Pythia QED-FSR PS or of PHOTOS. This dependence on the choice of the QED model is reduced after the matching of these tools with a fixed-order NLO-EW calculation: the differences, subleading in the counting of the final state QED mass logarithms, are pushed to $\mathscr{O}\left(\alpha^{2}\right)$, becoming numerically negligible.

\section{The role of the QCD modelling}

The above discussion assumes a given model to describe initial state QCD corrections; several choices have to be made to fully characterize the model: factorization and renormalization scales, the phase-space where the QCD-PS is allowed to generate radiation limited by a separation scale called scalup, the prescription to match fixed- and all-order results possibly parameterized by a separation scale ${ }^{1}$. Beside its perturbative content, the description of the DY process, and in particular of the lepton-pair transverse momentum distribution, depends: on the QCD PS internal parameters, including non-perturbative models to describe the intrinsic transverse momentum of the partons inside the proton, on the proton collinear PDF parameterization and the PDF experimental error on the different treatment necessary for the initial state heavy quarks, compared to the light-ones. An estimate of the uncertainties associated to all these parameters and their impact e.g. on the $W$ boson mass determination can be performed at an exploratory level considering the error propagation in the charged-current DY process alone (cfr. for instance the PDF studies in Refs. $[12,13])$, but a realistic estimate requires the inclusion of the correlation of these uncertainties among all the processes involved in the calibration of the detectors and Monte Carlo tools, in particular between the charged- and neutral-current DY channels.

The complex estimate of the different uncertainties mentioned above affects the QCD model on which the analysis of the charged current DY data is based and in turn also the interplay of QCD and EW corrections. We recall in fact that the size of the mixed QCDxEW effects has been estimated so far for a given QCD model [9], but that there is not an estimate of the uncertainties affecting these corrections, in particular of those stemming from the QCD modelling.

\section{Conclusions}

To summarize, while an estimate of the size of the QCDxEW corrections to the $W$ boson mass determination has been presented in Ref.[9], an estimate of the remaining theoretical uncertainties will require a global analysis of the QCD modelling of neutral- and charged-current DY processes on one side and the completion of the calculation of the exact $\mathscr{O}\left(\alpha \alpha_{s}\right)$ corrections to the partonic cross section on the other.

\footnotetext{
${ }^{1}$ In the Higgs boson case a detailed study of these issues has been presented in Refs.[10,11]
} 


\section{References}

[1] G. Aad et al. [ATLAS Collaboration], Eur. Phys. J. C 76 (2016) no.5, 291 doi:10.1140/epjc/s10052-016-4070-4 [arXiv:1512.02192 [hep-ex]].

[2] M. Aaboud et al. [ATLAS Collaboration], arXiv:1701.07240 [hep-ex].

[3] S. Alioli et al., Eur. Phys. J. C 77 (2017) no.5, 280 doi:10.1140/epjc/s10052-017-4832-7 [arXiv:1606.02330 [hep-ph]].

[4] S. Dittmaier, A. Huss and C. Schwinn, Nucl. Phys. B 885 (2014) 318 doi:10.1016/j.nuclphysb.2014.05.027 [arXiv:1403.3216 [hep-ph]].

[5] S. Dittmaier, A. Huss and C. Schwinn, PoS LL 2014 (2014) 045 [arXiv:1405.6897 [hep-ph]].

[6] S. Dittmaier, A. Huss and C. Schwinn, Nucl. Phys. B 904 (2016) 216 doi:10.1016/j.nuclphysb.2016.01.006 [arXiv:1511.08016 [hep-ph]].

[7] L. Barze, G. Montagna, P. Nason, O. Nicrosini and F. Piccinini, JHEP 1204 (2012) 037 doi:10.1007/JHEP04(2012)037 [arXiv:1202.0465 [hep-ph]].

[8] L. Barze, G. Montagna, P. Nason, O. Nicrosini, F. Piccinini and A. Vicini, Eur. Phys. J. C 73 (2013) no.6, 2474 doi:10.1140/epjc/s10052-013-2474-y [arXiv:1302.4606 [hep-ph]].

[9] C. M. Carloni Calame, M. Chiesa, H. Martinez, G. Montagna, O. Nicrosini, F. Piccinini and A. Vicini, arXiv:1612.02841 [hep-ph].

[10] E. Bagnaschi and A. Vicini, JHEP 1601 (2016) 056 doi:10.1007/JHEP01(2016)056 [arXiv:1505.00735 [hep-ph]].

[11] E. Bagnaschi, R. V. Harlander, H. Mantler, A. Vicini and M. Wiesemann, JHEP 1601 (2016) 090 doi:10.1007/JHEP01(2016)090 [arXiv:1510.08850 [hep-ph]].

[12] G. Bozzi, L. Citelli and A. Vicini, Phys. Rev. D 91 (2015) no.11, 113005 doi:10.1103/PhysRevD.91.113005 [arXiv:1501.05587 [hep-ph]].

[13] G. Bozzi, L. Citelli, M. Vesterinen and A. Vicini, Eur. Phys. J. C 75 (2015) no.12, 601 doi:10.1140/epjc/s10052-015-3810-1 [arXiv:1508.06954 [hep-ex]].

[14] R. Bonciani, S. Di Vita, P. Mastrolia and U. Schubert, JHEP 1609 (2016) 091 doi:10.1007/JHEP09(2016)091 [arXiv:1604.08581 [hep-ph]].

[15] R. Bonciani, F. Buccioni, R. Mondini and A. Vicini, Eur. Phys. J. C 77 (2017) no.3, 187 doi:10.1140/epjc/s10052-017-4728-6 [arXiv:1611.00645 [hep-ph]]. 